\title{
Wave Propagations in Nonlinear Low-Pass Electrical Transmission Lines through Optical Fiber Medium
}

\author{
Aniqa Zulfiqar, ${ }^{1}$ Jamshad Ahmad ${ }^{1},{ }^{1}$ Attia Rani, ${ }^{2}$ and Qazi Mahmood Ul Hassan ${ }^{2}$ \\ ${ }^{1}$ Department of Mathematics, University of Gujrat, Gujrat 50700, Pakistan \\ ${ }^{2}$ Department of Mathematics, University of Wah, Wah 47040, Pakistan \\ Correspondence should be addressed to Jamshad Ahmad; jamshadahmadm@gmail.com
}

Received 19 March 2021; Revised 2 April 2021; Accepted 16 December 2021; Published 5 January 2022

Academic Editor: Shahram Rezapour

Copyright (C) 2022 Aniqa Zulfiqar et al. This is an open access article distributed under the Creative Commons Attribution License, which permits unrestricted use, distribution, and reproduction in any medium, provided the original work is properly cited.

The present article discovers the new soliton wave solutions and their propagation in nonlinear low-pass electrical transmission lines (NLETLs). Based on an innovative Exp-function method, multitype soliton solutions of nonlinear fractional evolution equations of NLETLs are established. The equation is reformulated to a fractional-order derivative by using the Jumarie operator. Some new results are also presented graphically to understand the real physical importance of the studied model equation. The physical interpretation of waves is represented in the form of three-dimensional and contour graphs to visualize the underlying dynamic behavior of these solutions for particular values of the parameters. Moreover, the attained outcomes are generally new for the considered model equation, and the results show that the used method is efficient, direct, and concise which can be used in more complex phenomena.

\section{Introduction}

Considerable attention from scientists and researchers during the last two decades has highlighted that fractional differential simulations provide a better understanding than classical simulations to describe the complexities of physical scenarios in this real world. Various real life phenomena have been defined via the idea of fractional derivative such as porous medium, viscoelastic materials, optical fiber communication, fluid dynamics, signal processing, plasma physics, propagation of waves, ocean wave, photonic, electromagnetism, materials, chaotic systems, nanofiber technology, nuclear physics, and many others.

Currently, the most essential directions in the field of fractional calculus are to find the appropriate applications for describing the fractional operators. For this purpose, analytical and numerical techniques are used to produce the highly precise exact and approximate results. Recently, many authors have worked in this direction such as the efficient nonstandard finite difference scheme for a class of fractional chaotic systems [1]. A numerical technique has been used for resolving the fractional Hamilton equation with the help of
Caputo fractional operator [2], mathematical modelling of human liver with Caputo-Fabrizio fractional derivative [3], and mathematical model for Zika virus transmission [4]. Investigation of different types of soliton solutions of fractional differential equations (FDEs) can be identified through different techniques and has been examined by many authors [5-9]. Moreover, the application of fractionalorder differential equations has been clearly expressed by studying the hyperchaotic behaviors of a nonautonomous cardiac conduction system in both frames of integer and fractional-order differential equations [10].

Consequently, several techniques and methods, for example, the method of Painleve analysis [11], the method of Riemann-Hilbert [12], the technique of exp $(-\Phi(\xi))$-expansion [13], the technique of generalized Kudryashov [14], the technique of $\left(G^{\prime} / G, 1 / G\right)$-expansion [15], the trial solution approach [16], the Exp-function method [17], the modified simple equation method [18], and the technique of sine-Gordon expansion [19] are developed. $\mathrm{He}$ and $\mathrm{Wu}$ were the inventors of the Exp-function method [20] that was effectively extended to FDEs [21]. The Expfunction method has been applied to several nonlinear FDEs 
[22-34]. Recently, many authors worked on the new results of the fractional calculus [35]. Many well-known problems have been resolved by using the fractional derivatives such as p-Laplacian nonperiodic boundary value problem [36] and conformable Laplace's equation [37].

Transmission lines are utilized for the reason, such as high speed computer information buses, computer, arrange associations, trunk lines, steering calls between phone exchanging centers, dispersing cable tv signals, and interfacing radio transmitters and recipients with their receiving wires. The analytical solutions help in understanding the qualitative and quantity of many nonlinear phenomena and permit us to visualize the change mechanisms of various advanced nonlinear phenomena such as multiplicity or the absence of steady states below varied conditions, spatial localization of transfer processes, existence of peaking regimes, and lots of others. The examination of NLETLs and its soliton solution have spectacular applications in electronic engineering, fluid dynamics, geophysics, optical fiber, laser optics, and communication systems such as the television cable lines that allocate signals and many others. Furthermore, for the transmission of the alternate current of radio frequency signal, NLETLs are used as a special medium. The NLETLs are used to distribute the higher manner to specific the nonlinear excitation under nonlinear media and adjust the systems of recent structures. The NLETLs play crucial role in the investigation of propagation phenomena of electrical solitons where they are traveling in nonlinear media of dispersion within the form of voltage waves.

The NLETLs are derived with the help of Kirchoff's laws that have been seen in the study by Abdoulkary [38]. In preceding years, enormous research works have been done on NLETLs. Many researchers have been obtaining numerous kind of analytic solution with the help of different methods like Jacobi elliptic method, auxiliary equation method, tanh function method, Ricatti equation technique, Kudryashov technique and modified version of Kudryashov technique, the sine-Gorden method, and the extended form of sine-Gorden equation [39-42].

In this paper, our main purpose is to establish, in the fractional context, important results for NLETLs. For this purpose, the Exp-function method is used to resolve the nonlinear fractional evolution equation of NLETLs by using the modified Riemann-Liouville (RL) derivative that was proposed by Jumarie [43] for nonlinear fractional evolution equation of NLETLs. The modified RL derivative of order $\alpha$ is defined as follows:

$$
D_{t}^{\alpha} f(t)=\left\{\begin{array}{lr}
\frac{1}{\Gamma(1-\beta)} \frac{\mathrm{d}}{\mathrm{d} t} \int_{0}^{t}(t-\xi)^{-\alpha}(f(\xi)-f(0)) \mathrm{d} \xi, 0<\alpha<1, \\
\left(f^{n}(t)\right)^{(\alpha-n)}, & n \leq \alpha<n+1, n \geq 1 . \\
\text { graphs. To the best of our knowledge, } & u(x, t)=u(\eta), \\
\text { gravior for the presented model is } & \eta=k \frac{x^{\alpha}}{\alpha}+\omega \frac{t^{\alpha}}{\alpha},
\end{array}\right.
$$

where $k$ is the width of soliton and $\omega$ represents the velocity. Applying the transformation equation (2) reduces to the following ODE:

$$
R\left(u, u^{\prime}, u^{\prime \prime}, u^{\prime \prime \prime} \ldots\right)=0
$$

where the prime denotes the derivation with respect to $\eta$. If possible, we can integrate (4) one or more times. Suppose the soliton wave solution is

$$
u(\eta)=\frac{\sum_{m=-r}^{s} a_{m} \exp [m \eta]}{\sum_{n=-g}^{h} b_{n} \exp [n \eta]}
$$

Consider the general nonlinear FDEs as follows:

$$
\begin{aligned}
Q\left(u, u_{x}, u_{t}, D_{t}^{\alpha}, \ldots\right) & =0, \\
0 & <\alpha \leq 1 .
\end{aligned}
$$

In equation (2), $u$ is an unknown function, $Q$ represents the polynomial, $D_{t}^{\alpha}$ represents the fractional derivative, and several linear and nonlinear terms with higher-order derivative are also included. The fractional traveling wave transformation is implemented for transforming equation (2) into ODEs as represented in the following equation:

where $r, s, g$, and $h$ are positive integers which are unknown to be further determined and $a_{m}$ and $b_{n}$ are unknown constants. In equation (5), with the homogeneous balancing principle, the values of $r, s, \mathrm{~g}$, and $h$ will be determined.

\section{Numerical Application}

The nonlinear fractional evolution equation that describes the wave propagation in NLETLs is defined as follows [44]: 


$$
D_{t}^{2 \alpha} u+\beta D_{t}^{2 \alpha} u^{2}-d D_{t}^{2 \alpha} u^{3}+c^{2} \beta D_{x}^{2 \alpha} u^{2}+\frac{c^{4}}{12} D_{x}^{4 \alpha} u^{2}=0
$$

In equation (6), $\beta, d$, and $c$ are the real constants and $u(x, t)$ characterizes to transmission line voltage. $X$ signifies the distance, and $t$ symbolizes the slow time. For acquiring the soliton solutions for NLETLs, the Exp-function method will be applied to equation (6). For the conversion of equation (6) into an ordinary differential equation (ODE), we considered the fractional wave transformation as

$$
u(x, t)=u(\eta) \eta=k x^{\alpha} / \alpha+\omega t^{\alpha} / \alpha
$$

Substituting equation (3) in (6), we get

$$
12\left(k^{2} c^{2}+\omega^{2}\right) u+12 \beta \omega^{2} u^{2}-12 d \omega^{2} u^{3}+c^{4} k^{4} u^{\prime \prime}=0 .
$$

For simplicity, we set $r=s=g=h=1$, and then equation (5) is reduced to

$$
u(\eta)=\frac{a_{-1} \exp [-\eta]+a_{0}+a_{1} \exp [\eta]}{b_{-1} \exp [-\eta]+b_{0}+b_{1} \exp [\eta]}
$$

By substituting equation (9) in (8) and equating the coefficients of $\exp (n \eta)$ to zero, we find

$$
\begin{aligned}
& A_{1}=\left(12 b_{-1}^{2} a_{0}+24 a_{-1}\left(\beta a_{0}+b_{0}\right) b_{-1}+12 a_{-1}^{2}\left(\beta b_{0}-3 a_{0}\right)\right) \omega^{2} \\
& +c^{2} b_{-1} k^{2}\left(a_{0}\left(k^{2} c^{2}+12\right) b_{-1}-b_{0} a_{-1}\left(k^{2} c^{2}-24\right)\right)=0, \\
& A_{2}=\left(\begin{array}{c}
12 b_{-1}^{2} a_{1}+\left(24 b_{1} a_{-1}+24 a_{0} b_{0}+12 \beta\left(2 a_{-1} a_{1}+a_{0}^{2}\right)\right) b_{-1} \\
+12 a_{-1}\left(\beta a_{-1} b_{1}+2 \beta a_{0} b_{0}-3 a_{-1} a_{1}-3 a_{0}^{2}+b_{0}^{2}\right)
\end{array}\right) \omega^{2} \\
& +4 c^{2} k^{2}\left(a_{1}\left(k^{2} c^{2}+3\right) b_{-1}^{2}+\left(\begin{array}{c}
\left(-k^{2} c^{2} a_{-1}+6 a_{-1}\right) b_{1}- \\
\frac{a_{0} b_{0}\left(k^{2} c^{2}-24\right)}{4}
\end{array}\right) b_{-1}+\frac{a_{-1} b_{0}^{2}\left(k^{2} c^{2}+12\right)}{4}\right)=0 \\
& A_{3}=\left(\begin{array}{c}
\left(12 \beta a_{1}^{2}+24 b_{1} a_{1}\right) b_{-1}+12 b_{1}^{2} a_{-1}+\left(24 a_{0} b_{0}+12 \beta\left(2 a_{-1} a_{1}+a_{0}^{2}\right)\right) b_{1}+ \\
24 a_{1}\left(\beta a_{0} b_{0}-\frac{3}{2} a_{-1} a_{1}-\frac{3}{2} a_{0}^{2}+\frac{1}{2} b_{0}^{2}\right)
\end{array}\right) \omega^{2} \\
& -4 c^{2} k^{2}\left(\begin{array}{c}
a_{1} b_{1}\left(k^{2} c^{2}-6\right) b_{-1}+\left(-k^{2} c^{2} a_{-1}-3 a_{-1}\right) b_{1}^{2}-\frac{a_{0} b_{0}\left(k^{2} c^{2}-24\right) b_{1}}{4} \\
-\frac{a_{1} b_{0}^{2}\left(k^{2} c^{2}+12\right)}{4}
\end{array}\right)=0 \\
& A_{4}=\left(\begin{array}{c}
\left(24 b_{1} a_{0}+24 a_{1}\left(\beta a_{0}+b_{0}\right)\right) b_{-1}+24 a_{-1}\left(\beta a_{0}+b_{0}\right) b_{1}+12 a_{0} b_{0}^{2} \\
+12 \beta\left(2 a_{-1} a_{1}+a_{0}^{2}\right) b_{0}-12 a_{0}^{3}-72 a_{0} a_{-1} a_{1}
\end{array}\right) \omega^{2} \\
& -6 c^{2} k^{2}\left(\left(\left(k^{2} c^{2} a_{0}-4 a_{0}\right) b_{1}-\frac{a_{1} b_{0}\left(k^{2} c^{2}+8\right)}{2}\right) b_{-1}-\frac{b_{0}\left(a_{-1}\left(k^{2} c^{2}+8\right) b_{1}+4 a_{0} b_{0}\right)}{2}\right)=0, \\
& A_{5}=\left(12 b_{1}^{2} a_{0}+24 a_{1}\left(\beta a_{0}+b_{0}\right) b_{-1}+12 a_{1}^{2}\left(\beta b_{0}-3 a_{0}\right)\right) \omega^{2} \\
& +c^{2} b_{1} k^{2}\left(a_{0}\left(k^{2} c^{2}+12\right) b_{-1}-b_{0} a_{1}\left(k^{2} c^{2}-24\right)\right)=0, \\
& A_{6}=12 a_{1}\left(\left(\beta a_{1} b_{1}-a_{1}^{2}+b_{1}^{2}\right) \omega^{2}+k^{2} b_{1}^{2} c^{2}\right)=0, \\
& A_{7}=12 a_{-1}\left(\left(\beta a_{-1} b_{-1}-a_{-1}^{2}+b_{-1}^{2}\right) \omega^{2}+k^{2} b_{-1}^{2} c^{2}\right)=0 .
\end{aligned}
$$


By resolving the system of equations, we attain these where $a_{-1}, a_{1}$, and $b_{1}$ are free parameters. results.

Case1

$$
\begin{aligned}
\omega & =\frac{c b_{1} k}{\sqrt{-\beta a_{1} b_{1}+a_{1}^{2}-b_{1}^{2}}} \\
a_{-1} & =a_{-1}, \\
a_{0} & =0 \\
a_{1} & =a_{1}, \\
b_{-1} & =\frac{b_{1} a_{-1}}{a_{1}} \\
b_{0} & =0 \\
b_{1} & =b_{1},
\end{aligned}
$$

$$
u_{1}(x, t)=\frac{a_{-1} \exp \left[-K x^{\alpha} / \alpha-c b_{1} k t^{\alpha} / \alpha \sqrt{-\beta a_{1} b_{1}+a_{1}^{2}-b_{1}^{2}}\right]+a_{1} \exp \left[K x^{\alpha} / \alpha+c b_{1} k t^{\alpha} / \alpha \sqrt{-\beta a_{1} b_{1}+a_{1}^{2}-b_{1}^{2}}\right]}{b_{1} a_{-1} / a_{1} \exp \left[-K x^{\alpha} / \alpha-c b_{1} k t^{\alpha} / \alpha \sqrt{-\beta a_{1} b_{1}+a_{1}^{2}-b_{1}^{2}}\right]+b_{1} \exp \left[K x^{\alpha} / \alpha+c b_{1} k t^{\alpha} / \alpha \sqrt{-\beta a_{1} b_{1}+a_{1}^{2}-b_{1}^{2}}\right]}
$$

Case 2 where $a_{-1}, a_{0}$, and $b_{0}$ are free parameters.

$$
\begin{aligned}
\omega & =\frac{c b_{0} k}{\sqrt{-\beta a_{0} b_{0}+a_{0}^{2}-b_{0}^{2}}} \\
a_{-1} & =a_{-1}, \\
a_{0} & =a_{0}, \\
a_{1} & =0 \\
b_{-1} & =\frac{b_{0} a_{-1}}{a_{0}} \\
b_{0} & =b_{0}, \\
b_{1} & =0
\end{aligned}
$$

$$
u_{2}(x, t)=\frac{a_{-1} \exp \left[-K x^{\alpha} / \alpha-c b_{0} k t^{\alpha} / \alpha \sqrt{-\beta a_{0} b_{0}+a_{0}^{2}-b_{0}^{2}}\right]+a_{0}}{b_{0} a_{-1} / a_{0} \exp \left[-K x^{\alpha} / \alpha c b_{0} k t^{\alpha} / \alpha \sqrt{-\beta a_{0} b_{0}+a_{0}^{2}-b_{0}^{2}}\right]+b_{0}} .
$$


Case 3

$$
\begin{aligned}
\omega & =\frac{c b_{1} k}{\sqrt{-\beta a_{1} b_{1}+a_{1}^{2}-b_{1}^{2}}} \\
a_{-1} & =\frac{a_{1} b_{-1}}{b_{1}} \\
a_{0} & =a_{0}, \\
a_{1} & =a_{1}, \\
b_{-1} & =b_{-1}, \\
b_{0} & =\frac{b_{1} a_{0}}{a_{1}} \\
b_{1} & =b_{1},
\end{aligned}
$$

where $a_{1}, a_{0}, b_{-1}$, and $b_{1}$ are free parameters.

$$
u_{1}(x, t)=\frac{a_{1} b_{-1} / b_{1} \exp \left[-K x^{\alpha} / \alpha-c b_{1} k t^{\alpha} / \alpha \sqrt{-\beta a_{1} b_{1}+a_{1}^{2}-b_{1}^{2}}\right]+a_{0}+a_{1} \exp \left[K x^{\alpha} / \alpha+c b_{1} k t^{\alpha} / \alpha \sqrt{-\beta a_{1} b_{1}+a_{1}^{2}-b_{1}^{2}}\right]}{b_{-1} \exp \left[-K x^{\alpha} / \alpha-c b_{1} k t^{\alpha} / \alpha \sqrt{-\beta a_{1} b_{1}+a_{1}^{2}-b_{1}^{2}}\right]+b_{1} a_{0} / a_{1}+b_{1} \exp \left[K x^{\alpha} / \alpha+c b_{1} k t^{\alpha} / \alpha \sqrt{-\beta a_{1} b_{1}+a_{1}^{2}-b_{1}^{2}}\right]}
$$

Case 4 where $a_{1}, a_{0}$, and $b_{1}$ are free parameters.

$$
\begin{aligned}
\omega & =-\frac{c b_{1} k}{\sqrt{-\beta a_{1} b_{1}+a_{1}^{2}-b_{1}^{2}}} \\
a_{-1} & =\frac{a_{1} b_{-1}}{b_{1}} \\
a_{0} & =a_{0}, \\
a_{1} & =a_{1}, \\
b_{-1} & =b_{-1}, \\
b_{0} & =\frac{b_{1} a_{0}}{a_{1}} \\
b_{1} & =b_{1},
\end{aligned}
$$

$$
u_{4}(x, t)=\frac{a_{1} b_{-1} / b_{1} \exp \left[-K x^{\alpha} / \alpha+c b_{1} k t^{\alpha} / \alpha \sqrt{-\beta a_{1} b_{1}+a_{1}^{2}-b_{1}^{2}}\right]+a_{0}+a_{1} \exp \left[K x^{\alpha} / \alpha-c b_{1} k t^{\alpha} / \alpha \sqrt{-\beta a_{1} b_{1}+a_{1}^{2}-b_{1}^{2}}\right]}{b_{-1} \exp \left[-K x^{\alpha} / \alpha+c b_{1} k t^{\alpha} / \alpha \sqrt{-\beta a_{1} b_{1}+a_{1}^{2}-b_{1}^{2}}\right]+b_{1} a_{0} / a_{1}+b_{1} \exp \left[K x^{\alpha} / \alpha-c b_{1} k t^{\alpha} / \alpha \sqrt{-\beta a_{1} b_{1}+a_{1}^{2}-b_{1}^{2}}\right]}
$$


Case 5 where $a_{-1}, a_{1}$, and $b_{1}$ are free parameters.

$$
\begin{aligned}
\omega & =-\frac{c b_{1} k}{\sqrt{-\beta a_{1} b_{1}+a_{1}^{2}-b_{1}^{2}}}, \\
a_{-1} & =a_{-1}, \\
a_{0} & =0, \\
a_{1} & =a_{1}, \\
b_{-1} & =\frac{b_{1} a_{-1}}{a_{1}}, \\
b_{0} & =0, \\
b_{1} & =b_{1},
\end{aligned}
$$

$$
u_{5}(x, t)=\frac{a_{-1} \exp \left[-K x^{\alpha} / \alpha+c b_{1} k t^{\alpha} / \alpha \sqrt{-\beta a_{1} b_{1}+a_{1}^{2}-b_{1}^{2}}\right]+a_{1} \exp \left[K x^{\alpha} / \alpha-c b_{1} k t^{\alpha} / \alpha \sqrt{-\beta a_{1} b_{1}+a_{1}^{2}-b_{1}^{2}}\right]}{b_{1} a_{-1} / a_{1} \exp \left[-K x^{\alpha} / \alpha+c b_{1} k t^{\alpha} / \alpha \sqrt{-\beta a_{1} b_{1}+a_{1}^{2}-b_{1}^{2}}\right]+b_{1} \exp \left[K x^{\alpha} / \alpha-c b_{1} k t^{\alpha} / \alpha \sqrt{-\beta a_{1} b_{1}+a_{1}^{2}-b_{1}^{2}}\right]} .
$$

Case 6 where $a_{-1}, a_{0}$, and $b_{0}$ are free parameters.

$$
\begin{aligned}
\omega & =-\frac{c b_{0} k}{\sqrt{-\beta a_{0} b_{0}+a_{0}^{2}-b_{0}^{2}}}, \\
a_{-1} & =a_{-1}, \\
a_{0} & =a_{0}, \\
a_{1} & =0 \\
b_{-1} & =\frac{b_{0} a_{-1}}{a_{0}} \\
b_{0} & =b_{0}, \\
b_{1} & =0
\end{aligned}
$$

$$
u_{6}(x, t)=\frac{a_{-1} \exp \left[-K x^{\alpha} / \alpha+c b_{0} k t^{\alpha} / \alpha \sqrt{-\beta a_{0} b_{0}+a_{0}^{2}-b_{0}^{2}}\right]+a_{0}}{b_{0} a_{-1} / a_{0} \exp \left[-K x^{\alpha} / \alpha+c b_{0} k t^{\alpha} / \alpha \sqrt{-\beta a_{0} b_{0}+a_{0}^{2}-b_{0}^{2}}\right]+b_{0}} .
$$




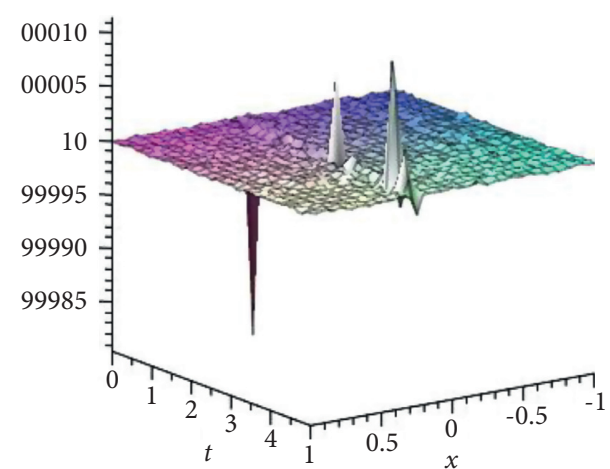

(a)

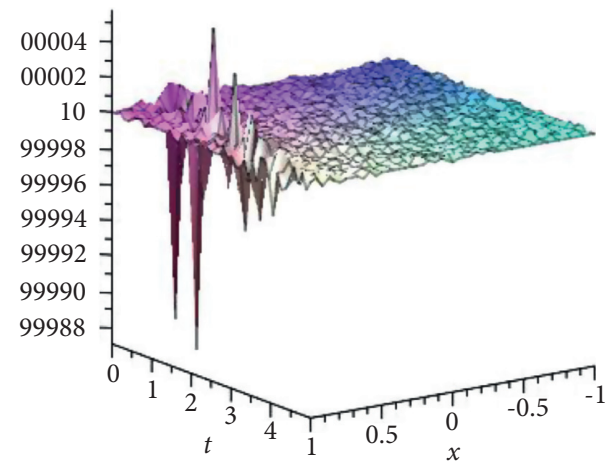

(c)

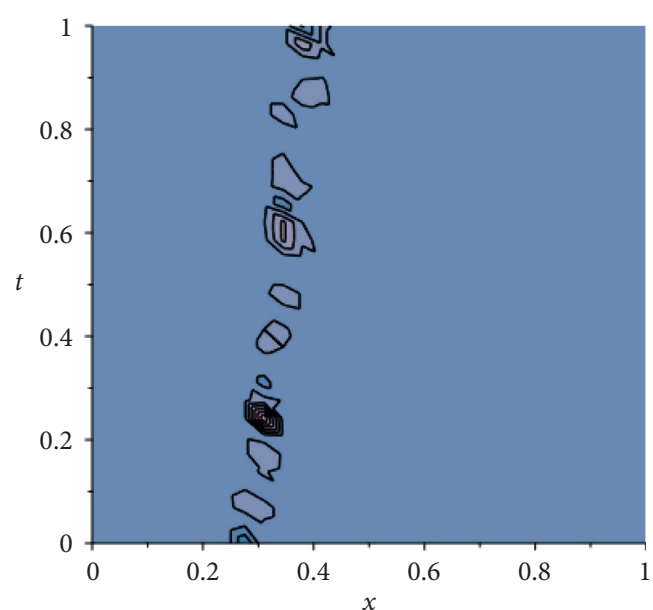

(e)

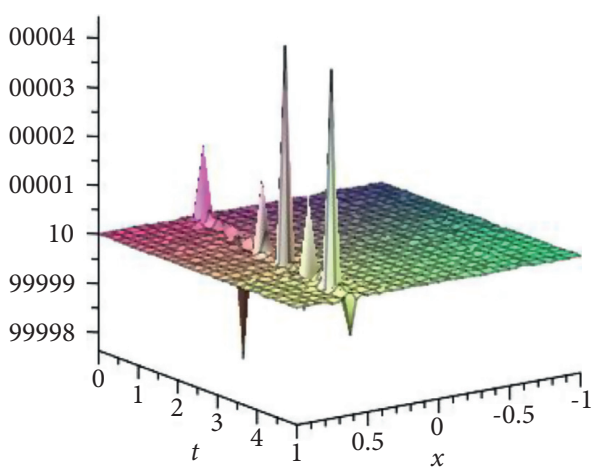

(b)

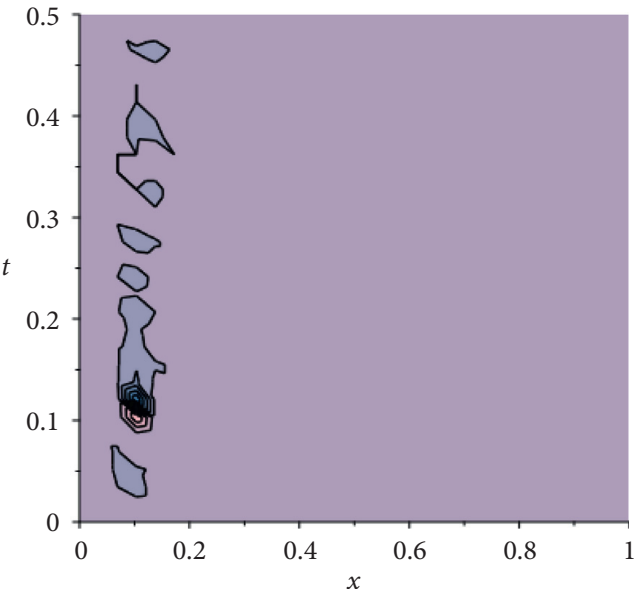

(d)

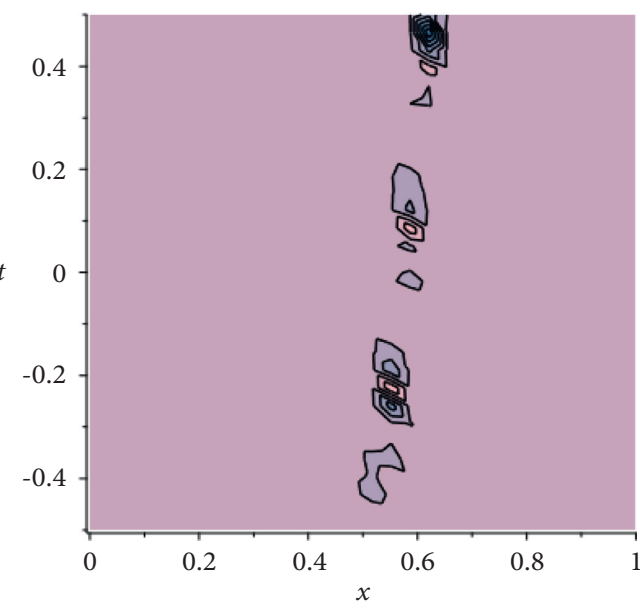

(f)

Figure 1: For $u_{1}(x, t),(\mathrm{a})-(\mathrm{c})$ with $-0.5 \leq x \leq 1,0 \leq t \leq 2$ signifying the $3 \mathrm{D}$ plots (d),(e), and (f) with $0 \leq x \leq 1,0 \leq t \leq 0.5 ; 0 \leq x \leq 1,0 \leq t \leq 1$; and $0 \leq x \leq 1,-0.5 \leq t \leq 0.5$, respectively, show the contour graph for different values of $\alpha$ : (a) $\alpha=0.5$; (b) $\alpha=0.7$; (c) $\alpha=1$; (d) $\alpha=0.5$; (e) $\alpha=0.7$; (f) $\alpha=1$. 


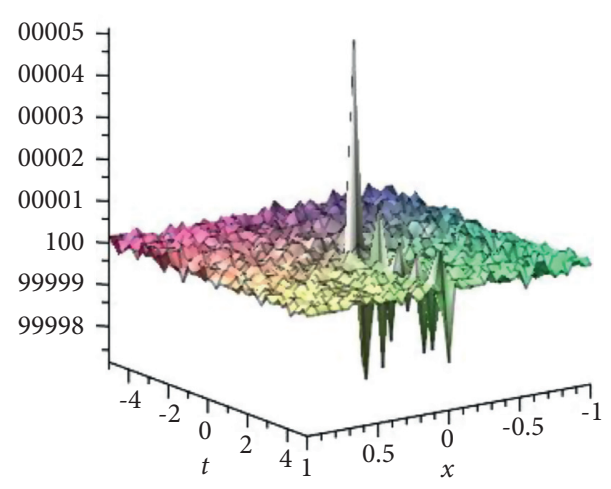

(a)

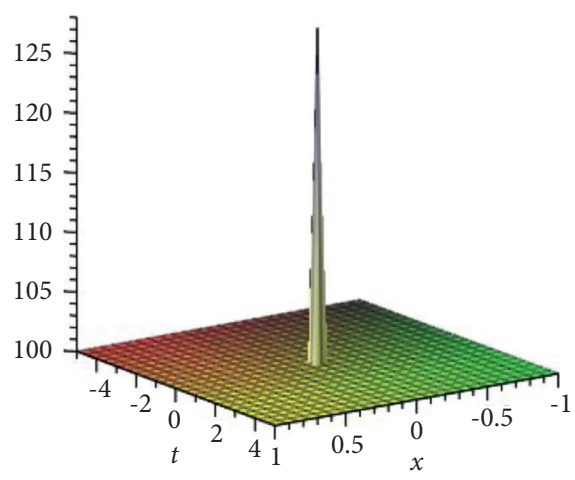

(c)

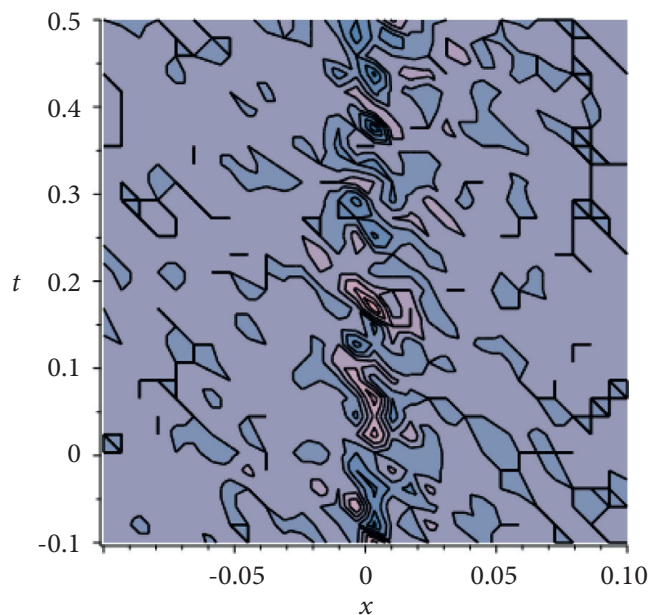

(e)

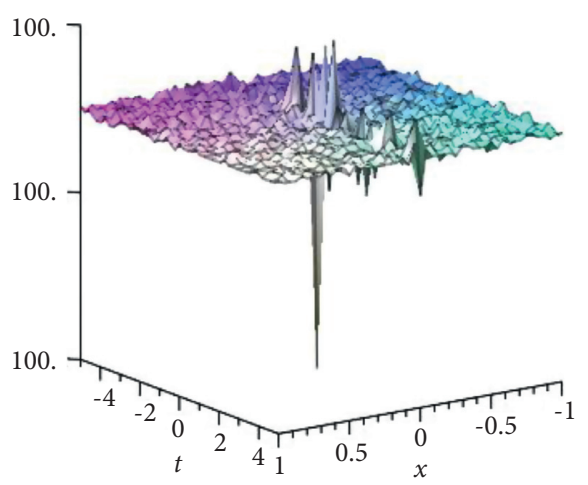

(b)

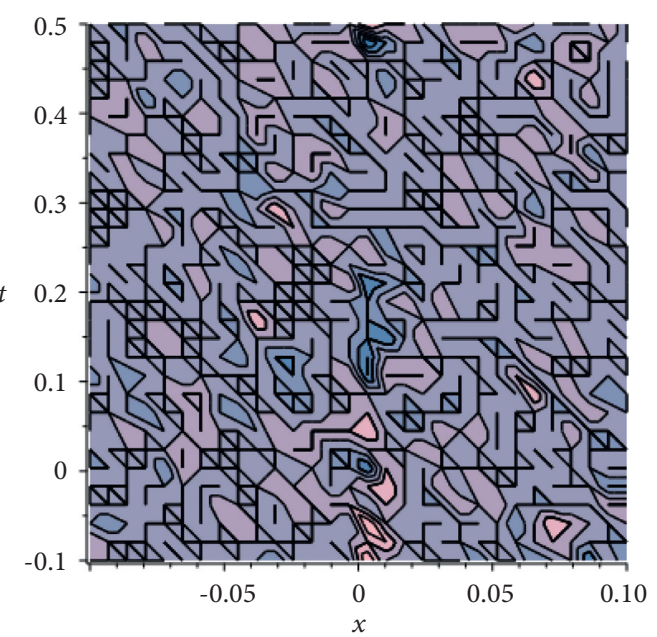

(d)

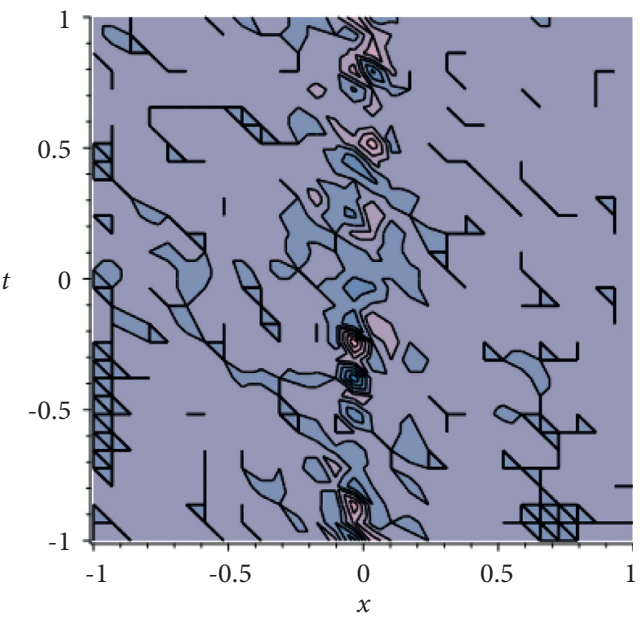

(f)

Figure 2: For $u_{2}(x, t)$, (a)-(c) with $-1 \leq x \leq 1,-5 \leq t \leq 5$ signifying the $3 \mathrm{D}$ plots (d), (e), and (f) with $-0.1 \leq x \leq 0.1,-0.1 \leq t \leq 0.5$; $-0.1 \leq x \leq 0.1,-0.1 \leq t \leq 0.5$; and $-1 \leq x \leq 1,-1 \leq t \leq 1$, respectively, show the contour graph for different values of $\alpha$ : (a) $\alpha=0.5$; (b) $\alpha=0.7$; (c) $\alpha=1$; (d) $\alpha=0.5$; (e) $\alpha=0.7$; (f) $\alpha=1$. 

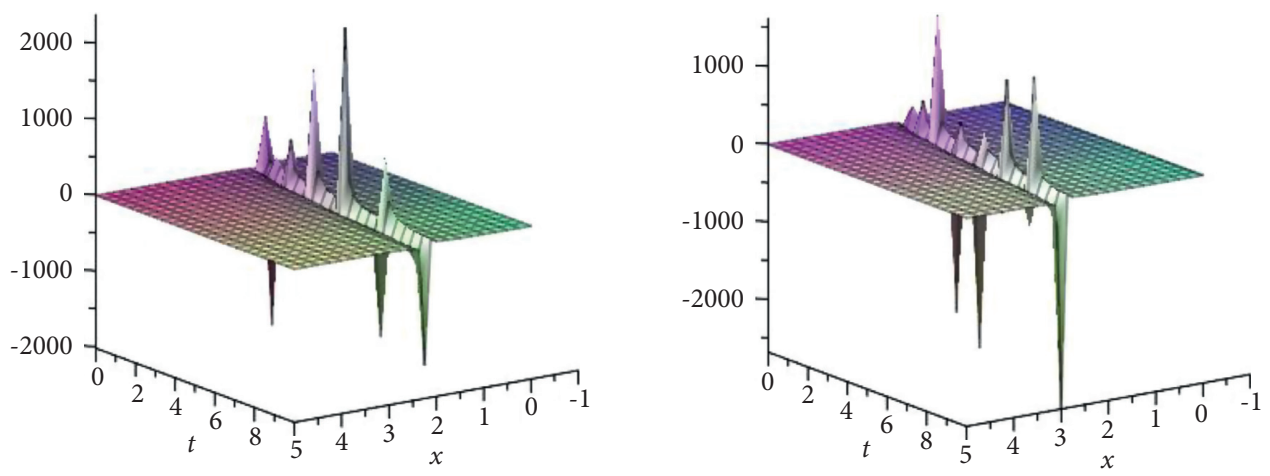

(a)

(b)
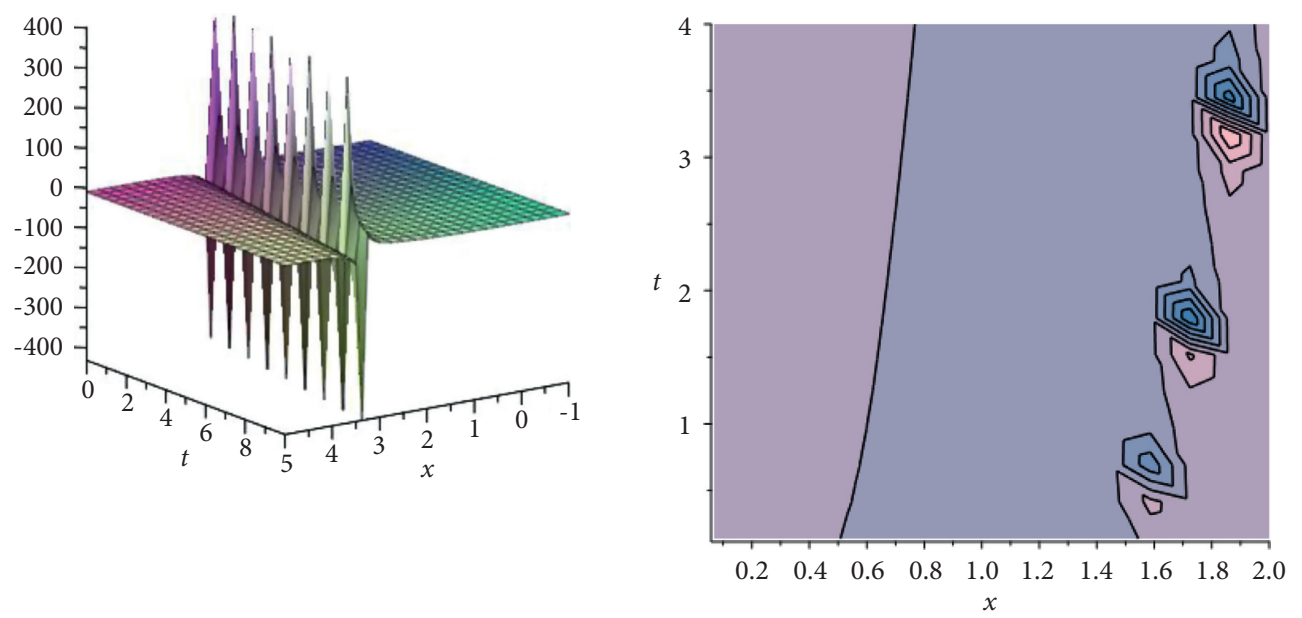

(c)

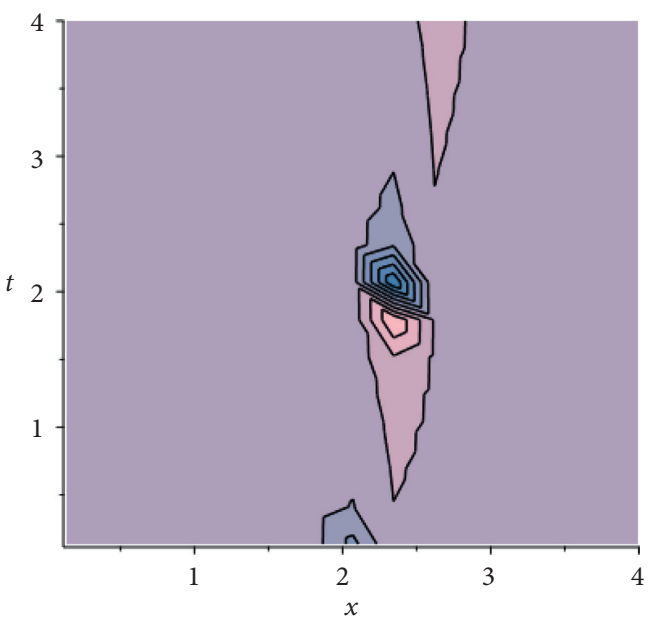

(d)

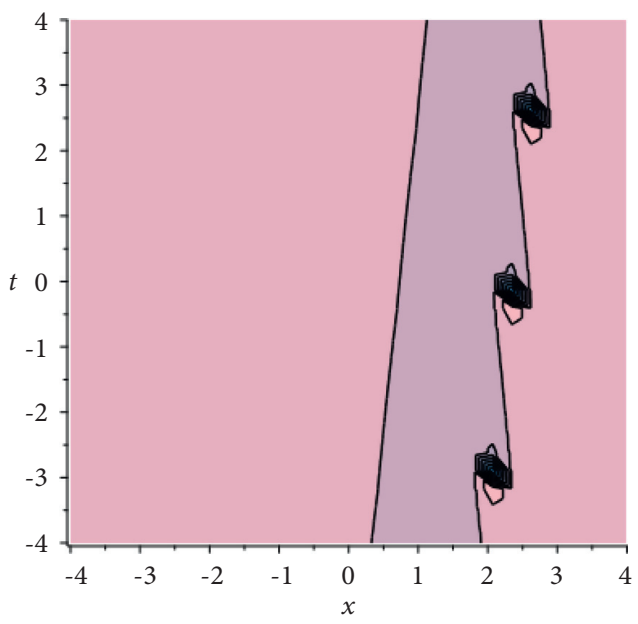

(f)

Figure 3: For $u_{3}(x, t)$, (a)-(c) with $-1 \leq x \leq 5,0 \leq t \leq 5$ signifying the $3 \mathrm{D}$ plots (d), (e), and (f) with $-2 \leq x \leq 2,-4 \leq t \leq 4$; $-4 \leq x \leq 4,-4 \leq t \leq 4$; and $-4 \leq x \leq 4,-4 \leq t \leq 4$, respectively, characterize the contour graph for different values of $\alpha$ : (a) $\alpha=0.5$; (b) $\alpha=0.7$; (c) $\alpha=1$; (d) $\alpha=0.5$; (e) $\alpha=0.7$; (f) $\alpha=1$. 


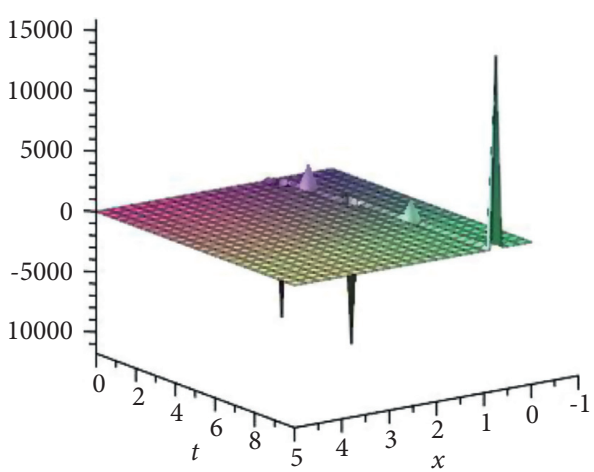

(a)

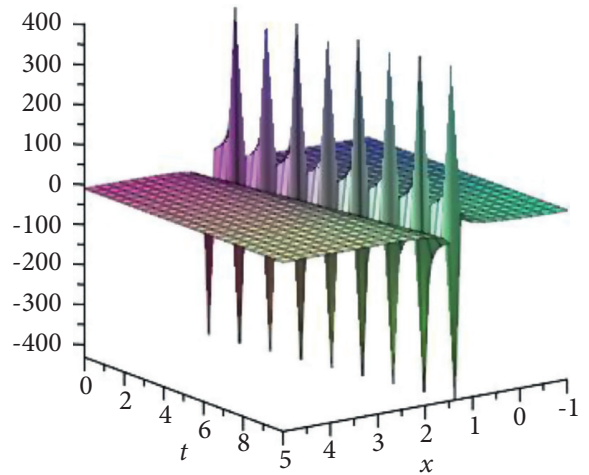

(c)

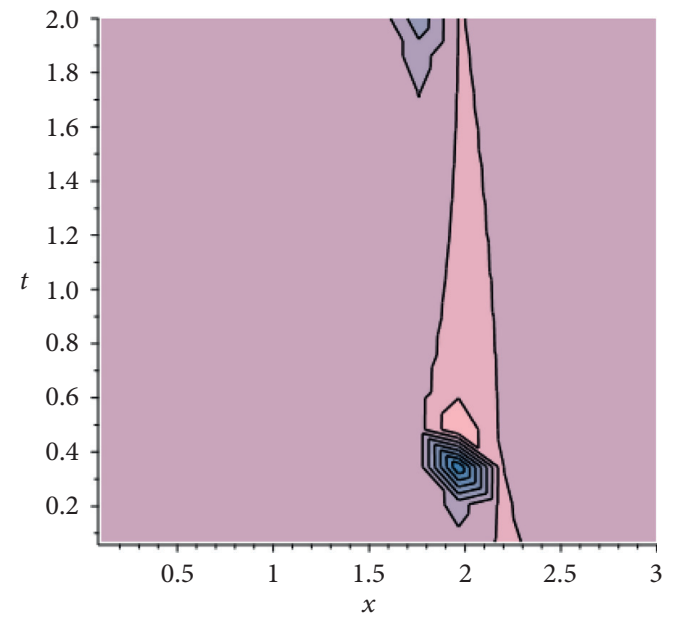

(e)

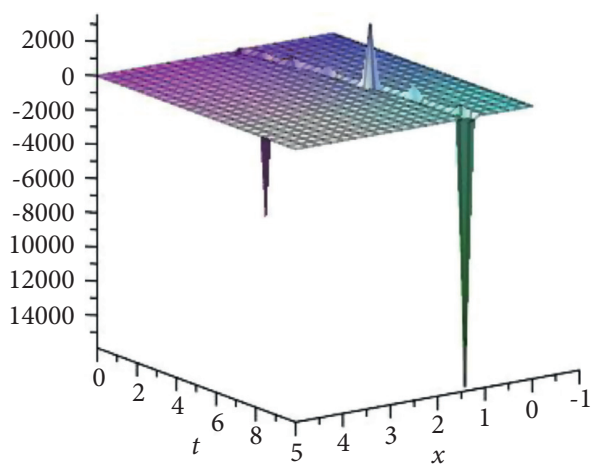

(b)

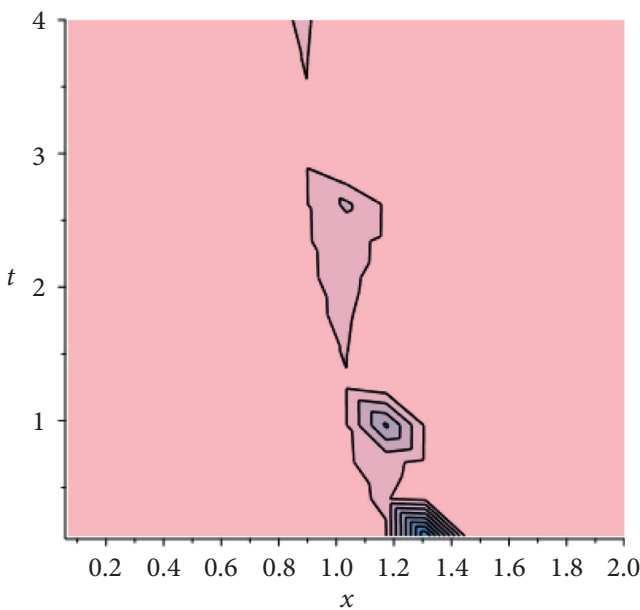

(d)

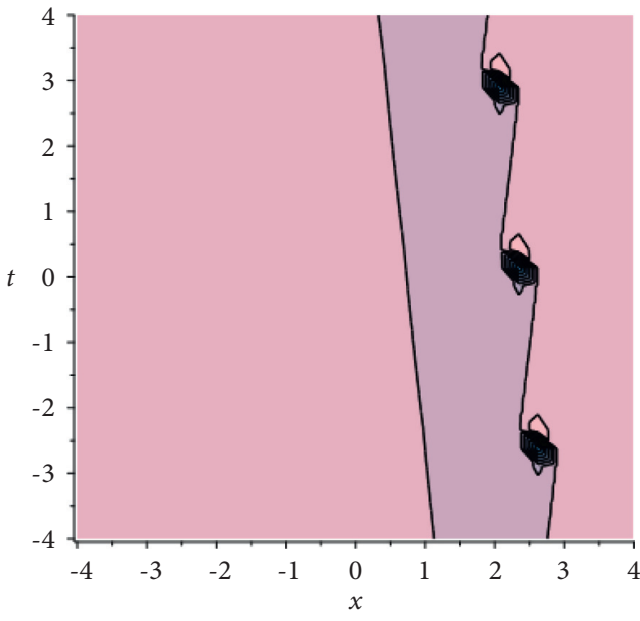

(f)

Figure 4: For $u_{4}(x, t)$, (a)-(c) with $-1 \leq x \leq 5,0 \leq t \leq 5$ characterizing the $3 \mathrm{D}$ plots, (d), (e), and (f) with $-2 \leq x \leq 2,-4 \leq t \leq 4$; $-3 \leq x \leq 3,-2 \leq t \leq 2$; and $-4 \leq x \leq 4,-4 \leq t \leq 4$, respectively, signify the contour graph for different values of $\alpha$ : (a) $\alpha=0.5$; (b) $\alpha=0.7$; (c) $\alpha=1$; (d) $\alpha=0.5$; (e) $\alpha=0.7$; (f) $\alpha=1$. 


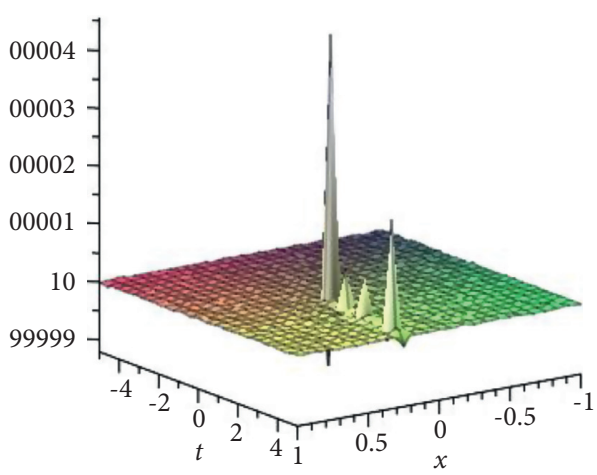

(a)

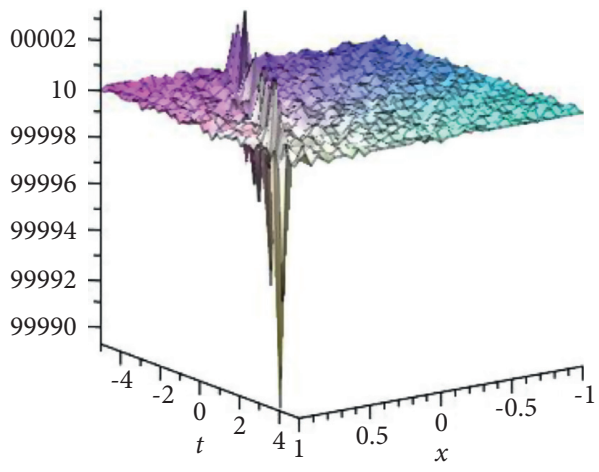

(c)

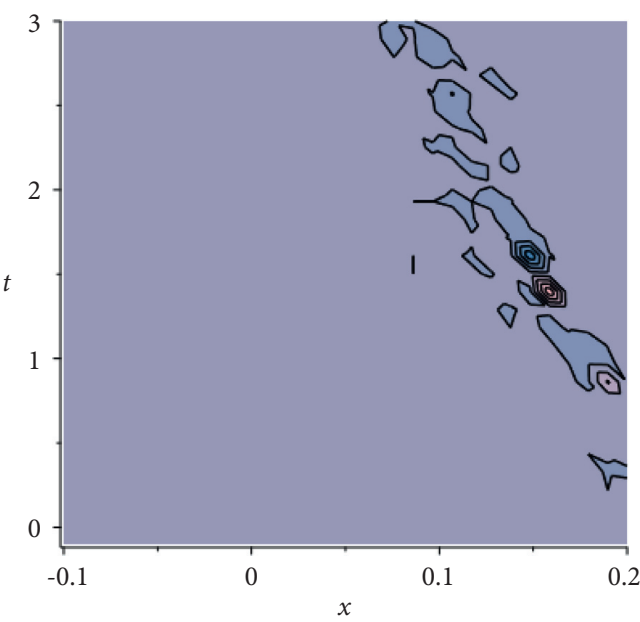

(e)

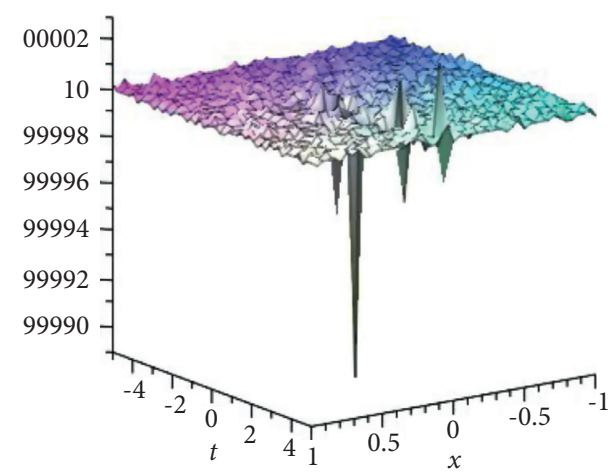

(b)

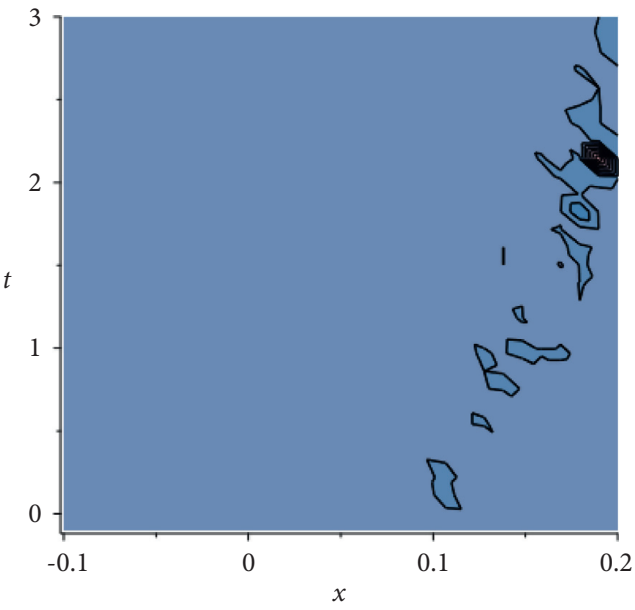

(d)

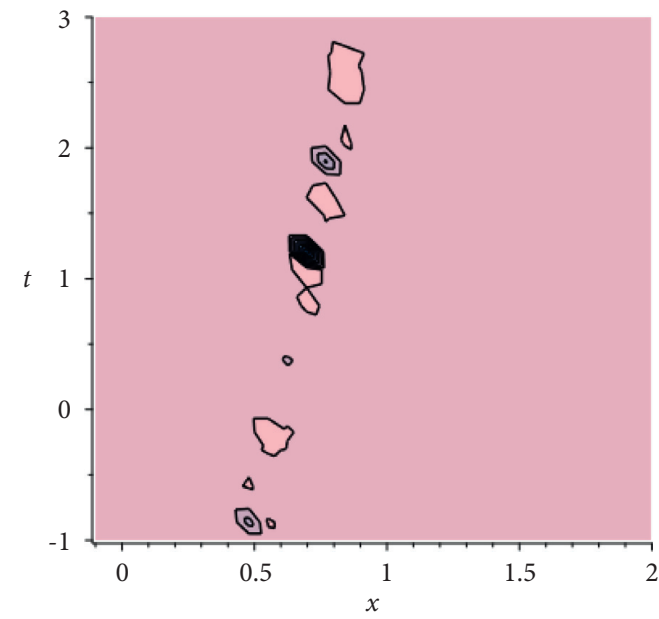

(f)

Figure 5: For $u_{5}(x, t)$, (a)-(c) with $-1 \leq x \leq 1,-5 \leq t \leq 5$ signifying the $3 \mathrm{D}$ plots (d), (e), and (f) with $-0.1 \leq x \leq 0.2,-0.1 \leq t \leq 3$; $-0.1 \leq x \leq 0.2,-0.1 \leq t \leq 3$; and $-0.1 \leq x \leq 2,-1 \leq t \leq 3$, respectively, indicate the contour graph for different values of $\alpha$ : (a) $\alpha=0.5$; (b) $\alpha=0.7$; (c) $\alpha=1$; (d) $\alpha=0.5$; (e) $\alpha=0.7$; (f) $\alpha=1$. 


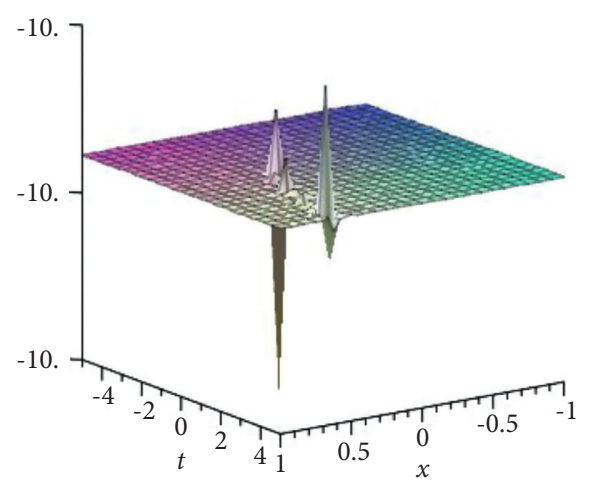

(a)

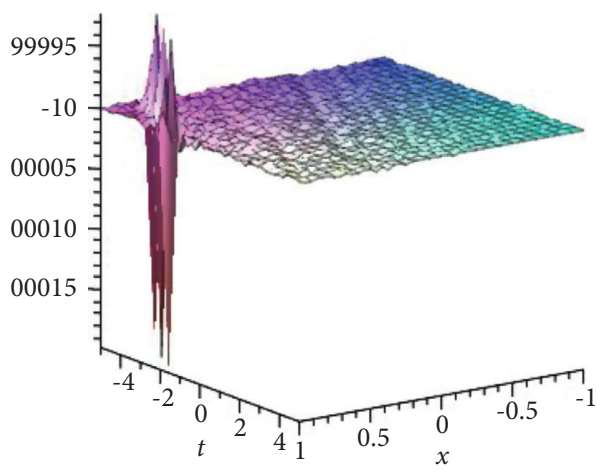

(c)

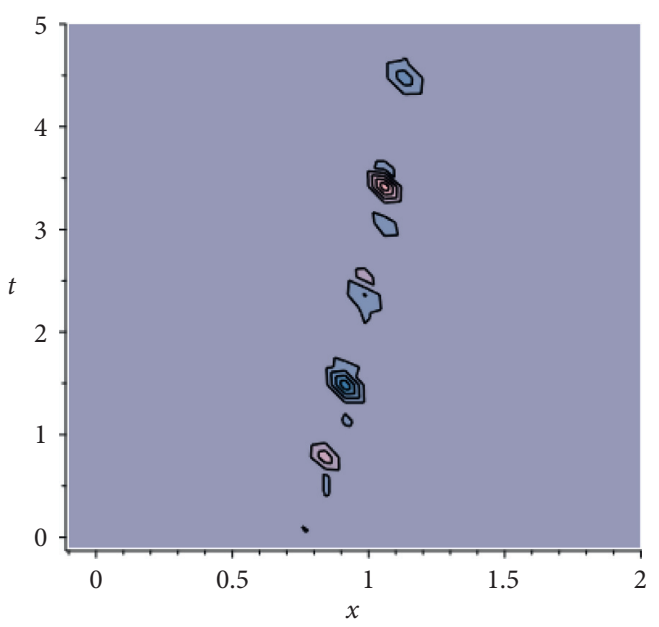

(e)

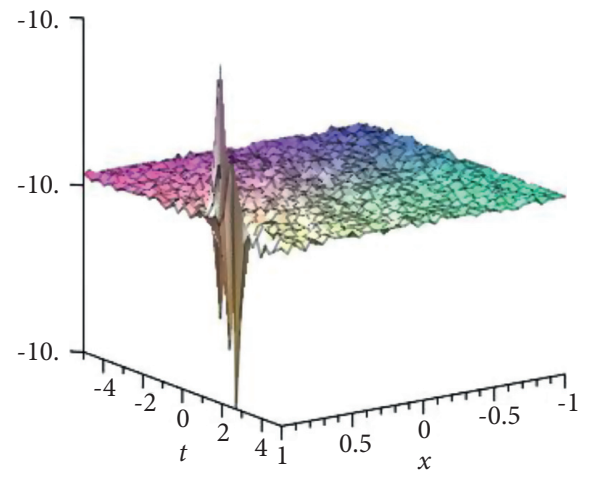

(b)

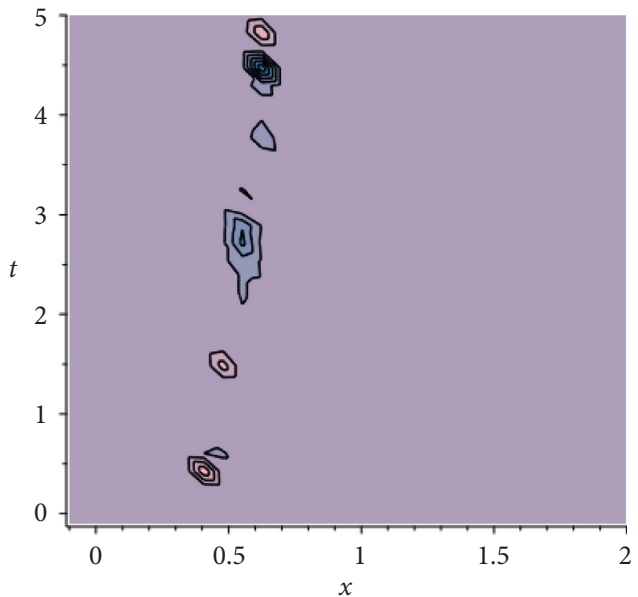

(d)

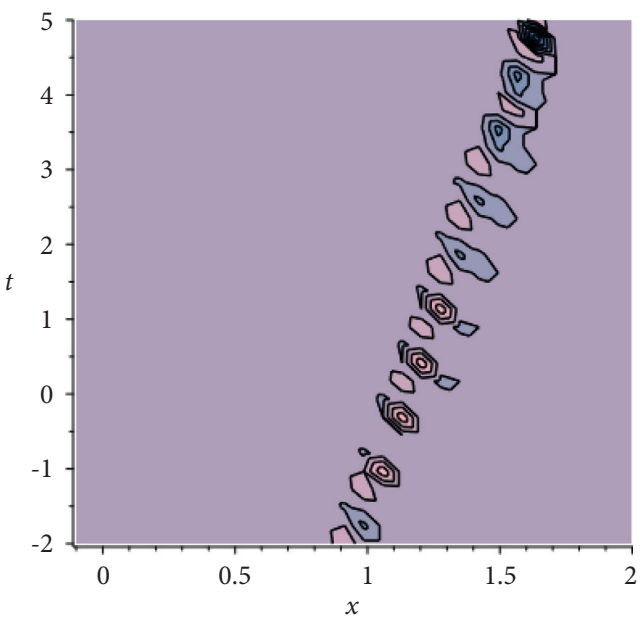

(f)

Figure 6: For $u_{6}(x, t)$, (a)-(c) with $-1 \leq x \leq 1,-5 \leq t \leq 5$ signifying the $3 \mathrm{D}$ plots (d), (e), and (f) with $-0.1 \leq x \leq 2,-0.1 \leq t \leq 5$; $-0.1 \leq x \leq 2,0.10 \leq t \leq 5$; and $-0.1 \leq x \leq 2,-2 \leq t \leq 5$, respectively, show the contour graph for different values of $\alpha$ : (a) $\alpha=0.5$; (b) $\alpha=0.7$; (c) $\alpha=1$; (d) $\alpha=0.5$; (e) $\alpha=0.7$; (f) $\alpha=1$. 


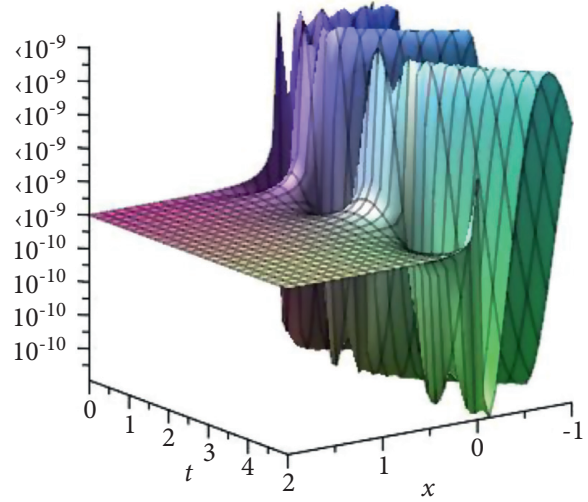

(a)

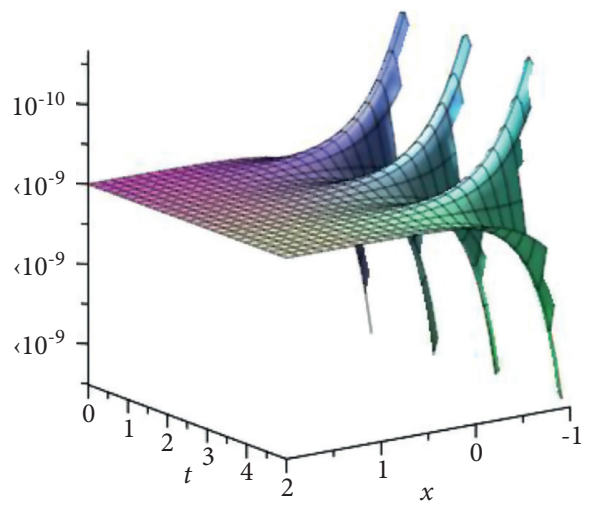

(c)

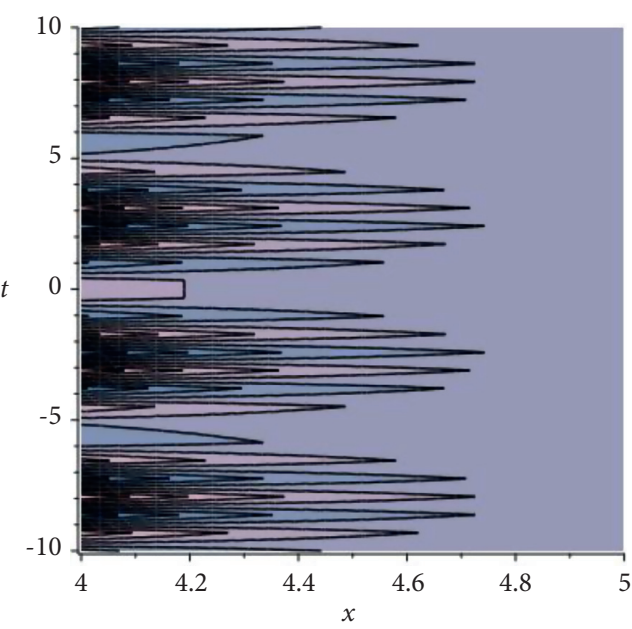

(e)

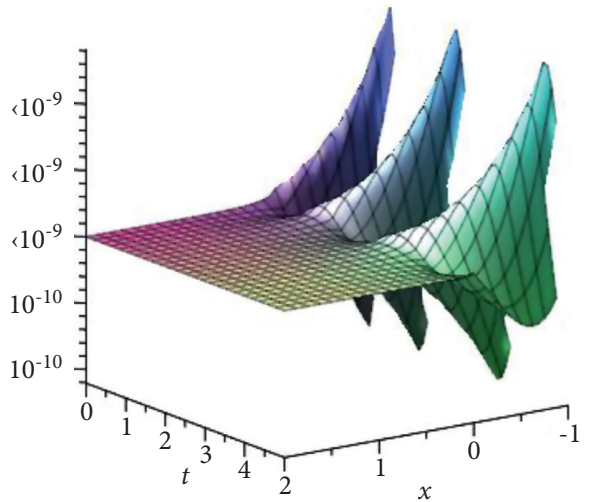

(b)

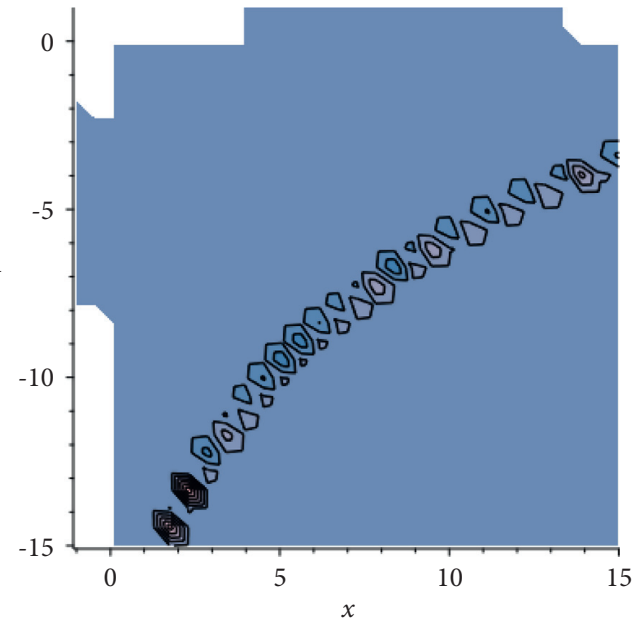

(d)

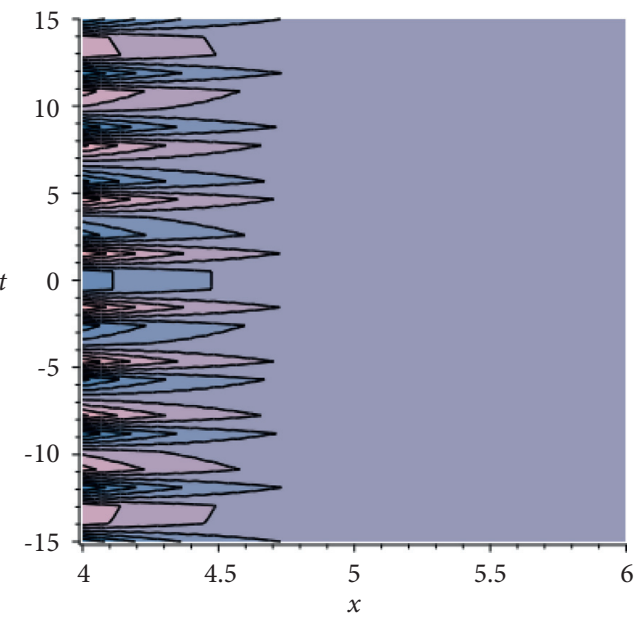

(f)

Figure 7: For $u_{7}(x, t)$, (a)-(c) with $-1 \leq x \leq 2,0 \leq t \leq 5$ signifying the $3 \mathrm{D}$ plots (d), (e), and (f) indicate the contour graph for $10 \leq x \leq 15,-15 \leq t \leq 15 ; 4 \leq x \leq 5,-10 \leq t \leq 10$; and $4 \leq x \leq 6,-15 \leq t \leq 15$, respectively, for different values of $\alpha$ : (a) $\alpha=0.5$; (b) $\alpha=0.7$; (c) $\alpha=1$; (d) $\alpha=0.5$; (e) $\alpha=0.7$; (f) $\alpha=1$. 
Case 7.

$$
\begin{aligned}
\omega & =-\frac{\sqrt{-3 c^{2} k^{2}-36 c k}}{6}, \\
a_{-1} & =0, \\
a_{0} & =a_{0}, \\
a_{1} & =0, \\
b_{-1} & =b_{-1}, \\
b_{0} & =-\frac{\beta a_{0}\left(k^{2} c^{2}+12\right)}{3 k^{2} c^{2}}, \\
b_{1} & =\frac{a_{0}^{2}\left(2 \beta^{2} k^{4} c^{4}+9 k^{4} c^{4}+48 \beta^{2} k^{2} c^{2}+108 k^{2} c^{2}+288 \beta^{2}\right)}{72 b_{-1} k^{4} c^{4}},
\end{aligned}
$$

where $a_{0}$ and $b_{-1}$ are free parameters.

$$
\begin{aligned}
u_{7}(x, t)= & \frac{a_{0}}{b_{-1} \exp \left[-K x^{\alpha} / \alpha+\sqrt{-3 c^{2} k^{2}-36 c k t^{\alpha}} / 6 \alpha\right]-\beta a_{0}\left(k^{2} c^{2}+12\right) / 3 k^{2} c^{2}} \\
& +a_{0}^{2}\left(2 \beta^{2} k^{4} c^{4}+9 k^{4} c^{4}+48 \beta^{2} k^{2} c^{2}+108 k^{2} c^{2}+288 \beta^{2}\right) / 72 b_{-1} k^{4} c^{4} \exp \left[K x^{\alpha} / \alpha-\sqrt{-3 c^{2} k^{2}-36 c k t^{\alpha}} / 6 \alpha\right]
\end{aligned}
$$

\section{Results and Discussion}

The erection of the soliton wave by determining nonlinear fractional evolution equation has been studied using the competent Exp-function method. We compare our acquired results with the previously existing solutions in the literature obtained by using different techniques. The main concentration of this work is to obtain new and more general solutions of fractional order at different values of parameters. In the literature, many different kinds of solutions have been obtained by using different techniques like dark, bright, trigonometric, hyperbolic, and rational type of solitary wave solutions as given in $[38,42,44]$.

Graphical data illustrate the physical behavior of solitons at different values of parameters. Figures 1-7 show the soliton solutions of NLETL equation of fractional order in the form of $3 \mathrm{D}$ and contour plots at $\alpha=0.4, \alpha=0.7$, and $\alpha=1$. Figure 1 signifies the physical behavior of $u_{1}(x, t)$ for $a_{-1}=0.1, \quad a_{1}=-0.01, b_{1}=-0.001, c=1, \beta=0.1$, and $\quad k=2$. Figure 2 signifies the plot of $u_{2}(x, t)$ at $a_{-1}=0.1, a_{0}=-0.1, b_{0}=-0.001, c=1, \beta=0.1$, and $k=2$. Figure 3 demonstrates the plot for $u_{3}(x, t)$ for $a_{-1}=0.1, a_{0}=$ $0.1, a_{1}=0.01, b_{-1}=1.5, b_{1}=-0.001, c=1, \quad \beta=0.1$, and $k=2$. Figure 4 characterizes the physical behavior of $u_{4}(x, t)$ for $a_{-1}=0.1, a_{0}=0.1, a_{1}=0.01, \quad b_{-1}=1.5, b_{1}=-0.001, c=1$, $\beta=0.1$, and $k=2$. Figure 5 denotes the plot of $u_{5}(x, t)$ with the parameter $a_{-1}=0.1, a_{1}=-0.01, b_{1}=-0.001, c=1, \beta=0.1$, and $k=2$. Figure 6 signifies the plot of $u_{6}(x, t)$ for $a_{-1}=0.1, a_{0}=-0.01, b_{0}=0.001, c=1, \beta=0.1$, and $k=2$. Figure 7 indicates the plot of $u_{7}(x, t)$ at $a_{0}=1 \times 10^{-12}, b_{0}=-0.00001, c=1, \beta=0.1$, and $k=3$.

We represented seven cases for different values of parameter using fractional derivative in this research work. It is clear from this work that fractional derivative has very important role in understanding the structure of the presented nonlinear evolution equation and also describes the continuous behavior of the solution wave through out the process. By comparing our results with the existing results, we conclude that our obtained results using the Exp-function method are generally new which have not been presented in the previous existing work. The complete examination proves that the presented technique is more consistent, competent, and dominant to examine different kinds of nonlinear fractional evolution equations.

\section{Conclusion}

In conclusion, the main attempt is to find and analyse the new soliton solutions of the fractional nonlinear fractional evolution equation that explains the wave propagation in nonlinear low-pass electrical transmission lines. The innovative Exp-function method with fractional traveling wave transform was utilized to obtain the well-known soliton solutions. Furthermore, from the graphical depiction, we have observed that different values of the parameters provide the different types of solutions for the low-pass electrical 
transmission lines. The solutions obtained from this model equation are applicable to various fields of science and engineering. Accordingly, we come to the conclusion that the presented model in this paper using the properties of fractional calculus is more flexible and genuinely analyzes real-world dynamical systems via the fractional-order differential operators from which the associated complicated dynamical behaviors of the problem can be revealed more precisely than the integer-order ones. Thus, it is well known that solitons have a great importance in the telecommunication system where the results obtained will probably help to carry information and increase the bit-rate of data. Also, we can extend our work for resolving the more complex biological and engineering problems using the properties and suitable operator of fractional calculus.

\section{Data Availability}

No data were used to support this study.

\section{Conflicts of Interest}

The authors declare that they have no conflicts of interest or personal relationships that could have appeared to influence the work reported in this paper.

\section{References}

[1] M. Hajipour, A. Jajarmi, and D. Baleanu, "An efficient nonstandard finite difference scheme for a class of fractional chaotic systems," Journal of Computational and Nonlinear Dynamics, vol. 13, no. 2, Article ID 021013, 2018.

[2] D. Baleanu, S. S. Sajjadi, A. Jajarmi, O. Defterli, and J. H. Asad, "The fractional dynamics of a linear triatomic molecule," Romanian Reports in Physics, vol. 73, no. 1, 2021.

[3] D. Baleanu, A. Jajarmi, H. Mohammadi, and S. Rezapour, "A new study on the mathematical modelling of human liver with Caputo-Fabrizio fractional derivative," Chaos, Solitons \& Fractals, vol. 134, Article ID 109705, 2020.

[4] S. Rezapour, H. Mohammadi, and A. Jajarmi, "A new mathematical model for Zika virus transmission," Advances in Difference Equations, vol. 2020, no. 1, 2020.

[5] V. E. Tarasov, Fractional Dynamics: Applications of Fractional Calculus to Dynamics of Particles, Fields and Media, Springer Science \& Business Media, Heidelberg, UK, 2011.

[6] S. Das, Functional Fractional Calculus, Springer Science \& Business Media, Heidelberg, UK, 2011.

[7] R. A. El-Nabulsi, "Path integral formulation of fractionally perturbed Lagrangian oscillators on fractal," Journal of Statistical Physics, vol. 172, no. 6, pp. 1617-1640, 2018.

[8] J. Dong and M. Xu, "Space-time fractional Schrödinger equation with time-independent potentials," Journal of Mathematical Analysis and Applications, vol. 344, no. 2, pp. 1005-1017, 2008.

[9] R. A. El-Nabulsi, "Emergence of quasiperiodic quantum wave functions in Hausdorff dimensional crystals and improved intrinsic Carrier concentrations," Journal of Physics and Chemistry of Solids, vol. 127, pp. 224-230, 2019.

[10] D. Baleanu, S. S. Sajjadi, J. H. Asad, A. Jajarmi, and E. Estiri, "Hyperchaotic behaviors, optimal control, and synchronization of a nonautonomous cardiac conduction system," Advances in Difference Equations, vol. 2021, no. 1, 2021.
[11] N. Farah, A. R. Seadawy, S. Ahmad, S. T. R. Rizvi, and M. Younis, "Interaction properties of soliton molecules and Painleve analysis for nano bioelectronics transmission model," Optical and Quantum Electronics, vol. 52, no. 7, pp. 1-15, 2020.

[12] T. Y. Xu, S. F. Tian, and W. Q. Peng, "Riemann-Hilbert approach for multisoliton solutions of generalized coupled fourth-order nonlinear Schrödinger equations," Mathematical Methods in the Applied Sciences, vol. 43, no. 2, pp. 865-880, 2020.

[13] S. Arshed, "Sub-pico second chirped optical pulses with TrikiBiswas equation by $\exp (-\Phi(\xi))$-expansion method and the first integral method," Optik, vol. 179, pp. 518-525, 2019.

[14] F. Mahmud, M. Samsuzzoha, and M. A. Akbar, "The generalized Kudryashov method to obtain exact traveling wave solutions of the PHI-four equation and the Fisher equation," Results in Physics, vol. 7, pp. 4296-4302, 2017.

[15] M. M. Miah, H. S. Ali, M. A. Akbar, and A. M. Wazwaz, "Some applications of the $\left(\mathrm{G}^{\prime} / \mathrm{G}, 1 / \mathrm{G}\right)$-expansion method to find new exact solutions of NLEEs," The European Physical Journal Plus, vol. 132, no. 6, pp. 1-15, 2017.

[16] M. Mirzazadeh, A. H. Arnous, M. F. Mahmood, E. Zerrad, and A. Biswas, "Soliton solutions to resonant nonlinear schrodinger's equation with time-dependent coefficients by trial solution approach," Nonlinear Dynamics, vol. 81, no. 1-2, pp. 277-282, 2015.

[17] A. Zulfiqar and J. Ahmad, "Soliton solutions of fractional modified unstable Schrödinger equation using Exp-function method," Results in Physics, vol. 19, Article ID 103476, 2020.

[18] M. A. Kayum, M. A. Akbar, and M. S. Osman, "Competent closed form soliton solutions to the nonlinear transmission and the low-pass electrical transmission lines," The European Physical Journal Plus, vol. 135, no. 7, pp. 1-20, 2020.

[19] K. K. Ali, A.-M. Wazwaz, and M. S. Osman, "Optical soliton solutions to the generalized nonautonomous nonlinear Schrödinger equations in optical fibers via the sine-Gordon expansion method," Optik, vol. 208, Article ID 164132, 2020.

[20] J. H. He, "Exp-function method for fractional differential equations," International Journal of Nonlinear Sciences and Numerical Stimulation, vol. 14, no. 6, pp. 363-366, 2013.

[21] O. Guner and A. Bekir, "Exp-function method for nonlinear fractional differential equations," Nonlinear Science Letters A, vol. 8, no. 1, pp. 41-49, 2017.

[22] J. M. Heris and M. Bagheri, "Exact solutions for the modified $\mathrm{KdV}$ and the generalized $\mathrm{KdV}$ equations via Exp-function method," Journal of Mathematical Extension, vol. 4, pp. 75-95, 2010.

[23] K. Ayub, M. Y. Khan, and Q. Mahmood-Ul-Hassan, "Solitary and periodic wave solutions of Calogero-BogoyavlenskiiSchiff equation via exp-function methods," Computers \& Mathematics with Applications, vol. 74, no. 12, pp. 3231-3241, 2017.

[24] S. T. Mohyud-Din, M. A. Noor, and K. I. Noor, "Exp-function method for traveling wave solutions of modified ZakharovKuznetsov equation," Journal of King Saud University Science, vol. 22, no. 4, pp. 213-216, 2010.

[25] M. A. Abdou, A. A. Soliman, and S. T. Basyony, "New application of Exp-function method for improved boussinesq equation," Physics Letters A, vol. 369, no. 5-6, pp. 469-475, 2007.

[26] S. A. El-Wakil, M. A. Madkour, and M. A. Abdou, "Application of exp-function method for nonlinear evolution equations with variable coefficient," Physics Letters A, vol. 369, no. 1-2, pp. 62-69, 2007. 
[27] M. A. Abdou, "New explicit exact solutions of nonlinear evolution equations using the generalized auxiliary equation method combined with Exp-function method," International Journal of Nonlinear Science, vol. 6, pp. 208-215, 2008.

[28] A. Zulfiqar and J. Ahmad, "Exact solitary wave solutions of fractional modified Camassa-Holm equation using an efficient method," Alexandria Engineering Journal, vol. 59, no. 5, pp. 3565-3574, 2020.

[29] X. H. Wu and J. H. He, "Solitary solutions, periodic solutions and compacton-like solutions using the Exp-function method," Computer Mathematical Application, vol. 54, no. 7-8, pp. 966-986, 2007.

[30] Y. Gurefe and E. Misirli, "Exp-function method for solving nonlinear evolution equations with higher order nonlinearity," Computers \& Mathematics with Applications, vol. 61, no. 8, pp. 2025-2030, 2011.

[31] O. Guner and A. Bekir, "The Exp-function method for solving nonlinear space-time fractional differential equations in mathematical physics," Journal of the Association of Arab Universities for Basic and Applied Sciences, vol. 24, no. 1, pp. 277-282, 2017.

[32] B. Zheng, "Exp-function method for solving fractional partial differential equations," The Scientific World Journal, vol. 2013, pp. 1-8, 2013.

[33] R. Rahmatullah, S. T. Mohyud-Din, and R. Khan, "Exact traveling wave solutions of fractional order Boussinesq-like equations by applying Exp-function method," Results in Physics, vol. 8, pp. 114-120, 2018.

[34] H. Ç. Yaslan and A. Girgin, "Exp-function method for the conformable space-time fractional STO, ZKBBM and coupled Boussinesq equations," Arab Journal of Basic and applied sciences, vol. 26, no. 1, pp. 163-170, 2019.

[35] F. Martínez, I. Martínez, M. K. Kaabar, and S. Paredes, "New results on complex conformable integral," AIMS Mathematics, vol. 5, no. 6, pp. 7695-7710, 2020.

[36] M. M. Matar, M. I. Abbas, J. Alzabut, M. K. A. Kaabar, S. Etemad, and S. Rezapour, "Investigation of the p-Laplacian nonperiodic nonlinear boundary value problem via generalized Caputo fractional derivatives," Advances in Difference Equations, vol. 2021, no. 1, pp. 1-18, 2021.

[37] F. Martínez, I. Martínez, M. K. Kaabar, and S. Paredes, "On conformable Laplace's equation," Mathematical Problems in Engineering, vol. 2021, Article ID 5514535, 10 pages, 2021.

[38] S. Abdoulkary, T. Beda, O. Dafounamssou, E. W. Tafo, and A. Mohamadou, "Dynamics of solitary pulses in the nonlinear low-pass electrical transmission lines through the auxiliary equation method," Journal of Modern Physics and Applications, vol. 2, pp. 69-87, 2013.

[39] M. Arshad, A. R. Seadawy, and D. Lu, "Modulation stability and dispersive optical soliton solutions of higher order nonlinear Schrödinger equation and its applications in monomode optical fibers," Superlattices and Microstructures, vol. 113, pp. 419-429, 2018.

[40] A. R. Seadawy, "Modulation instability analysis for the generalized derivative higher order nonlinear Schrödinger equation and its the bright and dark soliton solutions," Journal of Electromagnetic Waves and Applications, vol. 31, no. 14, pp. 1353-1362, 2017.

[41] İ. Aslan, "Exact solutions for a local fractional DDE associated with a nonlinear transmission line," Communications in Theoretical Physics, vol. 66, no. 3, pp. 315-320, 2016.

[42] I. Jaradat, M. Alquran, and K. Al-Khaled, "An analytical study of physical models with inherited temporal and spatial memory," The European Physical Journal Plus, vol. 133, no. 4, p. 162, 2018.

[43] G. Jumarie, "Modified Riemann-Liouville derivative and fractional Taylor series of nondifferentiable functions further results," Computers \& Mathematics with Applications, vol. 51, no. 9-10, pp. 1367-1376, 2006.

[44] A. R. Seadawy, M. Iqbal, and D. Baleanu, "Construction of traveling and solitary wave solutions for wave propagation in nonlinear low-pass electrical transmission lines," Journal of King Saud University Science, vol. 32, no. 6, pp. 2752-2761, 2020. 